\title{
Contents of Volume 33
}

\section{Number 1}

H. V. Klapdor: Beta Decay Far from Stability and Its Role in Nuclear Physics and Astrophysics . . . . . . . . . . . . . . . . . . . . . . . . . . . 1

\section{Number 2}

C. Edgar: Being Discrete about Yang and Mills: Basic Techniques of Euclidean Lattice Gauge Theory . . . . . . . . . . . . . . . . . . . . . . . . . . . . . . 57

L. L. DE RAAD, Jr.: Source Theory Treatment of the Casimir Effect: A Review . . . . . 117

\section{Number 3}

A. W. Hendry and D. B. Lichtenberg: Properties of Hadrons in the Quark Model . . . 139

\section{Number 4}

A. Gonzál Ez: Radiative Corrections to Bose Condensation . . . . . . . . . . . . . . . 233

M. Nishiok A : Weyl's Gauge Field and Its Behavior . . . . . . . . . . . . . . . . . . . 241

H. SATZ: The Limits of Hadron Thermodynamics . . . . . . . . . . . . . . . . . . . 259

\section{Number 5}

D. K. Grech : About Group Representations in Grand Unified Theories . . . . . . . . . 269

Ya. Z. Darbaidze, A. N. Sissakian, L. A. Slepchenko, H. T. Torosian: Analysis of Experimental Semi-Inclusive Distributions at the SPS-Collider-Energies . . . . . . . . . . 299

\section{Number 6}

A. O. BARUt, S. Komy: Derivation of Nonperturbative Relativistic Two-Body Equations from the Action Principle in Quantumelectrodynamics . . . . . . . . . . . . . . . 309

A. O. BardT, N. Únal: Radial Equations for the Relativistic Two-Fermion Problem with the Most General Electric and Magnetic Potentials . . . . . . . . . . . . . . . . . 319

A. Actor: Temperature Dependence of the $C P^{N-1}$ Model and the Analogy with Quantum Chromodynamics . . . . . . . . . . . . . . . . . . . . . . . . . . . 333

\section{Number 7}

M. KLEIN : Interference of Electromagnetic and Weak Interactions at High Energies and Neutral-Current $\mu e$ Universality 


\section{Number 8}

K. Westrfahl: High-Speed Scattering of Charged and Unoharged Particles in General Relativity . . . . . . . . . . . . . . . . 417

\section{Number 9}

V. A. Smirnov : Feynman Amplitudes as Tempered Distributions . . . . . . . . . 495

S. A. Aniris, V. A. Smirnov: Renormalization and Operator Product Expansion in Theories with Massless Particles . . . . . . . . . . . . . . . . . . . . . . . . . . 523

\section{Number 10}

W. Lucha: Proton Decay in Grand Unified Theories . . . . . . . . . . . . . . . 547

\section{Number 11/12}

V. I. Komarov, H. MülLer, S. Tesch: Cluster Excitation in Hadron-Nucleus Collisions at Medium and High Energies . . . . . . . . . . . . . . . . . . . . . . 595

H. J. Haubold, A. M. Mathat: The Maxwell-Boltzmannian Approach to the Nuclear Reaction Rate Theory . . . . . . . . . . . . . . . . . . . . . . 623

J. MaHarana: Canonical Structure and Quantization of Grassmann Variables . . . . . 645 